\title{
Cervical Cancer Prevention and Control Program in Cambodia: outcomes and contributing factors from a pilot implementation of cancer screening with the human papilloma virus test
}

\section{Kanal Koum}

Royal Government of Cambodia Ministry of Health

Noriko Fujita ( $\nabla$ norikof@it.ncgm.go.jp )

National Center for Global Health and Medicine https://orcid.org/0000-0002-2432-0987

Chan Soeung Sann

Royal Government of Cambodia Ministry of Health

\section{Leangsim Kruy}

Royal Government of Cambodia Ministry of Health

\section{Yasuyo Matsumoto}

Kokuritsu Kenkyu Kaihatsu Hojin Kokuritsu Kokusai Iryo Kenkyu Center

Rei Haruyama

Kokuritsu Kenkyu Kaihatsu Hojin Kokuritsu Kokusai Iryo Kenkyu Center

Kouji Banno

Keio Gijuku Daigaku

\section{Tadashi Kimura}

Osaka Daigaku Daigakuin Igakukei Kenkyuka Igakubu

\section{Research article}

Keywords: Cervical cancer, screening, Human Papilloma virus test, low-middle income countries, treatment of precancerous lesion, professional society

Posted Date: August 2nd, 2019

DOl: https://doi.org/10.21203/rs.2.12292/v1

License: (c) (1) This work is licensed under a Creative Commons Attribution 4.0 International License. Read Full License 


\section{Abstract}

Background Screening and treatment of precancerous lesions are not accessible to the majority of women in low-resource settings. Recently, the human papilloma virus (HPV) test has become affordable with its higher accuracy and more objective endpoint as a screening method; however, its implementation has been observed in a few resource-limited countries. This paper aims to describe the process of the pilot implementation of HPV test-based cervical cancer screening and treatment in Cambodia by Cambodian and Japanese societies of gynecology and to identify factors contributing to optimal outcomes. Methods A mixed-methods approach was used. Data from the survey results, the review of peer-reviewed and gray literature, and focus group discussions were analyzed. Results Implementation was performed in three phases: phase A to raise awareness of women's health and access to cervical cancer screening among factory workers; phase B to improve gynecologic capacity for the diagnosis and treatment of precancerous lesions; and phase $C$ to strengthen the capacity for pathological diagnosis. In phase A, among 687 eligible women, HPV test-based mobile screening was implemented for 132 women with $15 \mathrm{HPV}$ positives. In phase B, gynecologists at three national hospitals became capable of colposcopy and loop electrosurgical excision procedures using standardized protocols. In phase $\mathrm{C}$, the quality of technical skills and communication with clinical services improved for pathologists and technicians. Partnership between two professional societies, strong ownership and commitment of Cambodian society, and a comprehensive and stepwise quality-focused approach are identified to have contributed to the optimal outcomes and as key factors in sustainability. Conclusion Our analysis shows that HPV test-based screening has a potential to be scaled up with self-sustainability in Cambodia. This may serve as a good example of how professional societies can contribute to capacity building and system development for the prevention and control of cancer in a low-income country.

\section{Background}

Globally, an estimated 570,000 women are newly diagnosed with cervical cancer, and 311,000 women die every year, with approximately $90 \%$ of the cases occurring in low- and middle-income countries. $\left.{ }^{1}\right]$ Since the main cause of cervical cancer is the persistent infection of high-risk subtypes of human papillomavirus (HPV), this cancer is largely preventable through primary prevention with the HPV vaccine and health education as well as secondary prevention with the screening and treatment of precancerous lesions. [2] Indeed, cervical cancer incidence and mortality rates have decreased substantially over the last 30 years in high-income countries, mainly owing to the implementation of nationwide screening

programs . [2] However, these effective primary and secondary prevention measures are still not accessible to the majority of women in low-resource settings, and cervical cancer is identified only at an advanced stage, resulting in a high associated rate of death in these countries.

In Cambodia, with a female population of 8.2 million, cervical cancer is the most common cancer in women. The age-standard incidence rate (23.8 per 100,000 women) and mortality rate (13.4 per 100,000 
women) are much higher than the regional and global estimates. $\left[{ }^{3}\right]$ With an urgent need for action, cervical cancer was elevated to a disease to be given priority in the National Strategy for the Prevention and Control of Non-communicable Diseases (NCDs) 2007-2010.[4] In 2008, the Cambodian Ministry of Health $(\mathrm{MOH})$ issued a national guideline for cervical cancer screening with visual inspection with acetic acid (VIA) and immediate treatment with cryotherapy (i.e., "screen and treat") as the nationwide strategy. In the following national plan of NCDs 2013-2020, this approach was also listed as one of the "best-buy" interventions costing less than $\$ 1$ per person per year, $\left[{ }^{[}\right]$and the country aims to target over $60 \%$ of women aged 30-49 years to be screened at least once by the year 2020 .

Despite these efforts, the "screen and treat" strategy was still barely accessible to most women in Cambodia in 2015. Pilot programs were run with the support of different nongovernment organizations (NGOs), in which VIA was used for screening at clinics and health centers, with the referral of screen positives to district hospitals for cryotherapy. $\left.{ }^{[6,7}\right]$ Immediate treatment was associated with difficulty in procurement of supplies (e.g., liquid nitrogen). None of the programs were successful in scaling up due to the lack of collaboration in harmonizing clinical practice. Recently, the HPV test has become more affordable with its higher accuracy and more objective endpoint than other screening methods (i.e., VIA or cytology). $\left[^{8}\right]$ However, its implementation at the national level is observed only in a few resource-limited countries. $\left[^{9}\right]$

The Cambodian Society of Genecology and Obstetrics (SCGO) is the only society of professional obstetricians and gynecologists in Cambodia, established in 1997, with approximately 180 members as of 2015. With a request from the MOH, the SCGO was eager to address the issue of limited access to screening and treatment but needed technical support. The SCGO and the Japan Society of Obstetrics and Gynecology (JSOG) initiated a collaborative project in 2015 with the aim of establishing a workable and self-sustainable model for cervical cancer screening and the treatment of precancerous lesions.

This paper describes the process of the pilot implementation of HPV test-based cervical cancer screening and treatment of precancerous lesions at the national level in Cambodia and aims to identify factors that contributed to optimal outcomes. Since the call for action to end the suffering caused by cervical cancer by the Director General of the World Health Organization (WHO) in May 2018, countries and partners have been working towards the elimination of cervical cancer. Considering the experience in a resource-limited country is valuable to support the development of global and national strategies.

\section{Methods}


A mixed-methods approach was used to describe the process and examine factors that influenced the implementation process and contributed to optimal outcomes. Documents and reports in both published and gray literature in English, Khmer, and Japanese were reviewed. Databases (PubMed and Google Scholar) were searched for peer-reviewed literature using key search terms such as cervical cancer, health education, screening, treatment, and HPV test to identify all relevant papers. Gray literature includes policy documents and survey reports, minutes of regular project meetings, newsletters and reports of seminars and trainings in both Cambodia and Japan. In the last quarter of the project phases A and B described below, focus group discussions (15 SCGO and 4 JSOG members) and key informant interviews (4 SCGO and 2 JSOG members, 2 factory managers and 10 factory workers) were conducted to identify lessons learned, future challenges, and factors for optimizing outcomes (Additional file 1). After consent was obtained, notes were taken during interviews. Key words and sentences were extracted, coded and analyzed by coauthors until saturation was achieved. Extracted factors and lessons were triangulated with objective information from document review.

Project design and activities were approved by the $\mathrm{MOH}$ in Cambodia. The Ethical Board at the National Center for Global Health and Medicine, Japan, indicated that the study was exempt from ethical clearance because of the lack of human subjects.

\section{Overview of the project approach}

This project was designed based on the WHO's guide to Comprehensive Cervical Cancer Control.[2] It can be categorized into three project phases. Phase A (2015-2018) aimed to raise awareness of women's health and access to cervical cancer screening among factory workers; phase B (2015-2018) aimed to improve gynecologic capacity for the diagnosis and treatment of precancerous lesions; and phase $\mathrm{C}$ (2017-present) aimed to strengthen the pathological capacity for cancer diagnosis.

The project team consisted of SCGO and JSOG members, headed by the first and last author of this paper. SCGO gynecologists at three national referral hospitals were identified as core implementers of the pilot project. JSOG gynecologists and pathologists took part as technical advisors. As the target group for screening, factory workers were selected because of the rapid increase in the number and the social demand for their health and welfare in Cambodia.[10] A group of factories in the Phnom Penh Special Economic Zone was selected because of the easy access and monitoring by the SCGO and JSOG team.

\section{Results}

\section{Key interventions and primary outcomes}

Table 1 summarizes the characteristics of each phase, and Table 2 presents the key educational and managerial interventions conducted at the individual, organizational, and systematic levels. Figure 1 is a 
conceptual framework to show guidance in implementing activities, describing how the outcomes of the project are envisioned to help reduce the burden of cervical cancer in Cambodia.

\section{1) Phase A (Health education and screening)}

To assess the baseline knowledge, attitude, and practice of the target population regarding reproductive health and cervical cancer, a cross-sectional survey was conducted in Factory A in March 2016. Among 443 female workers, their level of knowledge of women's health and cervical cancer was low. Approximately $85 \%$ reported that their sources of information were relatives or friends, indicating that they rarely have opportunities to receive accurate knowledge from health professionals. $\left[{ }^{11}\right]$ Based on their educational needs, teaching materials and brochures on cervical cancer were developed.

\section{2) Phase $B$ (Diagnosis and treatment of precancerous lesions)}

Situational analysis to examine the current practice at the target hospitals in November 2015 found that machines for diagnosis (colposcopy) and treatment of precancerous lesions (loop electrosurgical excision procedures (LEEP)) were already installed in all three hospitals but were often used improperly. Records regarding the number of abnormal cases detected and treated were poorly recorded. $\left[{ }^{15}\right]$

To improve the skill of colposcopy and LEEP, a series of hands-on trainings was conducted both on-site and in Japan between 2015 and 2018. LEEP was favored over cryotherapy for the treatment of precancerous lesions because it allows the lesions to be pathologically examined for definitive diagnosis and improves surgical capacity at tertiary hospitals responsible for cancer care. To harmonize practice, a standard clinical protocol was developed to guide clinical decision-making, and it was reviewed and revised after a year. Reporting forms and a registry for HPV-positive cases were also developed for monitoring.

\section{3) Phase C (Pathology service)}

Through phase $B$, the obstacle to scaling up a cervical cancer screening program in Cambodia was identified as the extremely limited capacity of pathological services. When any screening program is scaled up in a country, there will inevitably be a large increase in the detection rate of cervical cancer. In 2017, however, there were only four pathologists and 15 pathology technicians actively working in the whole country. The situation was even worse than in 2014 because of the retirement of older generations. $\left[{ }^{17}\right]$ 
At the target hospitals, although basic pathology equipment and supplies were available, slides were often difficult to read due to inadequate preparation. A step-by-step training was offered to technicians for quality slide preparation. Five pathology residents, enrolled in the national residency program that started in 2015 to increase the number of pathologists, were also trained for their capacity-building in diagnosis and quality management.

Under the initiative of the SCGO, a clinico-pathological conference (CPC) was introduced to improve case management. In the beginning, pathologists and gynecologists tended to criticize each other on discrepancies between clinical and pathological diagnoses, but with close mentoring by Japanese physicians, a mutual learning environment was soon created. The CPC has gradually become a routine practice for developing clinical management skills.

\section{Factors contributing to optimal outcomes}

The lessons learned and challenges identified in the focus group discussion are summarized in Table 3 . The following factors that influenced the implementation process and contributed to optimal outcomes were identified:

\section{1) Partnership between two professional societies}

Since 1998, JSOG members have been working with the Cambodian MOH through development projects. Individual collaboration became more institutional in 2012 when an exchange program was initiated between the JSOG and SCGO. The idea for this project came from the SCGO. Throughout the project implementation, the JSOG respected the initiative of the SCGO and adopted a coaching style to provide technical and managerial guidance as a professional society that has a public responsibility. The SCGO took this opportunity to develop institutional capacity and leadership and attracted over 300 physicians across the country in 2018.

\section{2) Strong ownership and commitment of the SCGO}

With a request from the $\mathrm{MOH}$ and as a professional society responsible for women's health, the SCGO took the initiatives and leadership in every step to introduce HPV test-based screening and treatment and 
capacity building for pathology services. Since many board members are retired or are presently highranking officials of the $\mathrm{MOH}$ with extensive clinical and administrative experience, negotiation with factory managers and national hospital directors or communication with the $\mathrm{MOH}$ and development partners was smooth and successful. In fact, there is no group in Cambodia other than the SCGO that is technically and professionally capable of responding to this issue.

\section{3) A comprehensive and stepwise quality-focused approach with stakeholder involvement}

Quality is essential for cancer care. The stepwise quality-focused approach taken in this project revealed opportunities and barriers in each step of the comprehensive cancer care continuum. Solutions to address the barriers were discussed and responded to on time.

In phase A, combined cervical cancer with sexuality and women's health was revealed to be best for communicating with women and factory managers. Women showed considerable interest in learning about their own health along with the factory managers, who considered the session to be useful in maintaining a healthy workforce. Factory managers gradually became cooperative with the project, their awareness of cervical cancer increased, and mobile screening became feasible at factory.

In phase $\mathrm{B}$, the involvement of national hospital directors from the initial stage made maximum utilization of existing resources possible. Finally, HPV test-based screening and treatment of precancerous lesions were introduced within a relatively small budget (Table 1). For the future development of the cancer care continuum, cervical cancer programs may serve as an entry point for strengthening pathology services in a country.

\section{4) Socioeconomic factors}

Increased demand for social welfare and quality health care pushed the SCGO to address cervical cancer as a priority agenda and to initiate this project. The National Health Insurance Scheme, introduced in January 2018, enabled formal workers to seek consultation and receive necessary treatment without copayment. Public bus service and Uber-type application service have been expanded around the city of Phnom Penh, with improved geographical access to hospitals.

\section{Discussion}

This paper describes a pilot implementation of a program for HPV test-based screening and treatment of precancerous lesions as well as an effort to strengthen pathological capacity in the country. A 
complementary role and joint society initiatives could contribute to moving activities forward. Achieving a goal as a joint activity between professional societies is a somewhat novel approach. Their moral authority as national professional societies can lead to building both ownership and leadership. This approach could be a key factor in sustainability for developing the system of cervical cancer management.

The WHO recommends a comprehensive approach to cervical cancer prevention and control that includes multidisciplinary interventions throughout the life course: community education, social mobilization, vaccination, screening, treatment, and palliative care. [2] In Cambodia, the HPV vaccine demonstration program started in 2017 targeting 9-year-old girls through a school-based approach in six districts.[18] With a coverage rate of $94 \%$, the $\mathrm{MOH}$ plans to integrate the HPV vaccine into the national immunization program by $2020 .\left[^{19}\right]$ In parallel with vaccination, a nationwide screening program and cancer management system must be implemented. An alternative approach to VIA for screening, now the HPV test, has the potential for scaling up in Cambodia.

For future scaling up of screening, one challenge is the follow-through from health education to screening and from screening to follow-up examination. Even though screening was conducted on-site under the support of factory managers and free of charge, the participation rate was low (19.2\%). This contrasts with a literature review reporting that educational intervention increases women's participation in screening, particularly when the literacy level is low in the target communities. $\left[^{8}\right]$ Even though further analysis of nonparticipation is needed, possible reasons for nonparticipation are being busy or tired on the day of screening and the unpleasantness of pelvic examinations. Self-sampling has been reported to have high acceptability and feasibility in recent literature and might be worth exploring.[20,21] For the follow-up evaluation, the call-recall system showed some effectiveness in our practice. To address the issue of the inability to be absent from work and transportation costs, the provision of follow-up evaluation and the treatment of precancerous lesions on-site could also be an option.

A wide-scale awareness campaign to create knowledge as well as demand for screening is usually used for scaling up interventions. Before the potential demand is created, service availability for screening, diagnosis and treatment, i.e., a capable workforce and functioning medical device, need to be in place. The fact that the HPV test became affordable-\$5.4 per test (excluding personal costs) -offers the potential to introduce HPV test-based screening nationwide. Assuming that HPV-negative women receive regular screening every 5 years as recommended, $\left[{ }^{2}\right]$ the annual cost of screening will become $\$ 1-2$ per woman, with much higher accuracy than VIA. Further cost analysis and modeling for scaling up are needed for financial planning. 
The small sample of interventions at the national level limits the reliability of application, especially with a capable workforce. The screening protocol developed in this project can be applied to other tertiary hospitals. However, it needs to be adapted for use at primary and secondary levels given resource constraints. Where colposcopy and LEEP are not available, smartphone image application and thermal ablation may be utilized. [22-25] In any circumstances, capacity building of service providers can be facilitated in a cascading manner by the SCGO under collaboration with the JSOG.

Regarding treatment, Cambodia faces an increasing cancer burden and rising demand for quality care. There is a need, as a first step, to establish a stable foundation for diagnostic pathology services. Before cervical cancer screening programs are introduced on a large scale, plans for cancer management systems, including pathology, surgery, radiotherapy and palliative care, should also be developed and organized.

\section{Conclusion}

Our analysis shows that there is potential for HPV test-based screening to be scaled up with selfsustainability, even though challenges are recognized and solutions discussed. Key contributing factors are based on joint society initiatives and a step-wise quality-focused approach. Two societies have a plan to continue working on the expansion of the comprehensive approach after 2019. This effort might serve as a good example of how professional societies can contribute to capacity building and system development for the prevention and control of cancer in a resource-limited country such as Cambodia.

\section{List Of Abbreviations}

CPC: Clinico-pathological conference

JSOG: the Japan Society of Obstetrics and Gynecology

HPV: Human papilloma virus

LEEP: Loop electrosurgical excision procedures

$\mathrm{MOH}$ : Ministry of Health

NCDs: Non-communicable Diseases

NGOs: Nongovernment organizations

SCGO: The Cambodian Society of Genecology and Obstetrics 
VIA: Visual inspection with acetic acid

WHO: World Health Organization

\section{Declarations}

\section{Ethics approval and consent to participate}

Overall project design and all activities in each phase were approved by the $\mathrm{MOH}$, Cambodia. A cross sectional survey conducted during the project in March 2016 was approved by the Ethical Committee of the $\mathrm{MOH}$, Cambodia. Following the approval of the $\mathrm{MOH}$, informed consent was obtained in a written form to participate cancer screening and treatment, and in a verbal form in interview. The Ethical Committee of the National Center for Global Health and Medicine, Japan, approved this procedure and indicated that the study was exempt from ethical clearance because of the lack of human subjects by using the secondary data from project activity reports.

\section{Consent for publication}

Not applicable

\section{Competing interests}

None declared

\section{Data Availability}

Data sharing is not applicable to this article as no datasets were generated or analysed during the current study.

\section{Funding}

Funding was obtained from Japan International Cooperation Agency (JICA) and Japanese Ministry of Health, Labor and Welfare. The funders have no role in the study design, data collection and analysis, decision to publish, or preparation of the manuscript.

\section{Authors' contribution}


KK, NF, CS, LK, and TK developed the project design and conceptualization, and prepared the funding proposal. KK and TK took the overall responsibility of the project implementation. All authors contributed to implement the project and analyze lessons. NF, RH, YM wrote the first draft, and all authors reviewed and agreed with the final version of the paper.

\section{Acknowledgement}

The authors acknowledge SCGO and JSOG members who participated in the project for their contribution and cooperation. Authors also acknowledge Prof. Gilbert Burnham, Johns Hopkins Bloomberg School of Public Health, for his support for elaborating the manuscript.

\section{References}

1. Ginsburg O, Bray F, Coleman MP, et al. The global burden of women's cancers: a grand challenge in global health. Lancet. 2017;389(10071):847-860.

2. World Health Organization. Comprehensive cervical cancer control: a guide to essential practice. WHO Library Cataloguing-in-Publication Data. 2014.

3. Ferlay J, Ervik M, Lam F, C et al. Global Cancer Observatory: Cancer Today. Lyon, France: International Agency for Research on Cancer. 2018. Available from: https://gco.iarc.fr/today, accessed 4 May, 2019.

4. Ministry of Health Cambodia. National Strategy for the Prevention and Control of Noncommunicable Disease 2007-2010.

5. Ministry of Health Cambodia. National Strategic Plan for the Prevention and Control of Noncommunicable Diseases 2013-2020.

6. L Loun C. National strategy for cervical cancer management and a pilot approach in Cambodia (VIACryotherapy). Presented at the Asia Pacific Scientific Workshop, 4 March 2019. Singapore

7. Thay S, Peprah SA, Hur C, et al. Prevalence of Cervical Dysplasia in HIV-Positive and HIV-Negative Women at the Sihanouk Hospital Center of HOPE, Phnom Penh , Cambodia. Asian Pac J Cancer Prev. 2019;20(2):653-659.

8. Mustafa RA, Santesso N, Khatib R, et al. Systematic reviews and meta-analyses of the accuracy of HPV tests, visual inspection with acetic acid, cytology, and colposcopy. Int J Gynaecol Obstet. 2016;132(3):259-265.

9. World Health Organization. Assessing National Capacity for the Prvention and Control of Noncommunicable Diseases: Report of the 2017 Global Survey. 2018. Available from: https://www.who.int/ncds/surveillance/ncd-capacity/en/, accessed 4 May, 2019.

10. United Nations Population Fund. Literature Review on Sexual and Reproductive Health and Rights of Migrant Garment Factory Workers in Cambodia. 2014. Available 
from: https://cambodia.unfpa.org/sites/default/files/pub-

pdf/SRHRofGarmentFactoryWorkerLiteratureReview_2.pdf, accessed 4 May, 2019.

11. Cambodian Society of Gynecology and Obstetrics. SCGO-JSOG Project - Women's Health and Cervical Cancer-Introduction of the Project. 2015. Available from: http://scgo-kh.com/projectactivities, accessed 4 May, 2019.

12. Kelly H, Mayaud P, Segondy M, Pant Pai N, Peeling RW. A systematic review and meta-analysis of studies evaluating the performance of point-of-care tests for human papillomavirus screening. Sex Transm Infect. 2017;93(S4):S36-S45.

13. Ueda Y, Kawana K, Yanaihara N, et al. Development and evaluation of a cervical cancer screening system in Cambodia: A collaborative project of the Cambodian Society of Gynecology and Obstetrics and Japan Society of Obstetrics and Gynecology. J Obstet Gynaecol Res. 2019;11(10):13968.

14. Schiffman M, Castle PE. The promise of global cervical-cancer prevention. N Engl J Med. 2005;353(20):2101-2104.

15. Kina UK, K Aun, K Soeung, et al. Current situation of cervical cancer diagnosis, management and registration in three national tertiary hospitals in Cambodia. April 2016. Preseneted at the 68th Annual Congress of the Japan Society of Obstetrics and Gynecology, Tokyo Japan

16. Ridsdale LL. Cervical screening in general practice: call and recall. $J$ R Coll Gen Pract. 1987;37(299):257-259.

17. Vathana CS, Stauch G. Pathology and telepathology in Cambodia. Pathology. 2014;46:S8.

18. Garon J. Preventing Cervical Cancer in Cambodia: Evaluating the HPV Vaccination Demonstration Project. 2018. Available from: https://blogs.cdc.gov/global/2018/04/24/preventing-cervical-cancerin-cambodia/, accessed 4 May, 2019.

19. Vichit O. Review of HPV vaccine demonstration program in Cambodia. May 2018. Presented at National Immunization Program Office, Phnom Penh, Cambodia

20. Kellen E, Benoy I, Vanden Broeck D, et al. A randomized, controlled trial of two strategies of offering the home-based HPV self-sampling test to non- participants in the Flemish cervical cancer screening program. Int J Cancer. 2018;143(4):861-868.

21. Modibbo F, Iregbu KC, Okuma J, et al. Randomized trial evaluating self-sampling for HPV DNA based tests for cervical cancer screening in Nigeria. Infect Agent Cancer. 2017;12:11.

22. Gallay $C$, Girardet A, Viviano M, et al. Cervical cancer screening in low-resource settings: a smartphone image application as an alternative to colposcopy. Int $J$ Womens Health. 2017;9:455461.

23. Tanaka Y, Ueda Y, Okazawa A, et al. 'Smartscopy' as an alternative device for cervical cancer screening: a pilot study. BMJ Innov. 2017;3(2):123-126.

24. Dolman L, Sauvaget C, Muwonge R, Sankaranarayanan R. Meta-analysis of the efficacy of cold coagulation as a treatment method for cervical intraepithelial neoplasia: a systematic review. BJOG. 2014;121(8):929-942. 
25. Cremer M, Paul P, Bergman K, Haas M, et al. A Non-Gas-Based Cryotherapy System for the Treatment of Cervical Intraepithelial Neoplasia: A Mixed-Methods Approach for Initial Development and Testing. Glob Health Sci Pract. 2017;5(1):57-64.

\section{Figures}

Figure 1. Concept map of the Cambodia Cervical Cancer Project

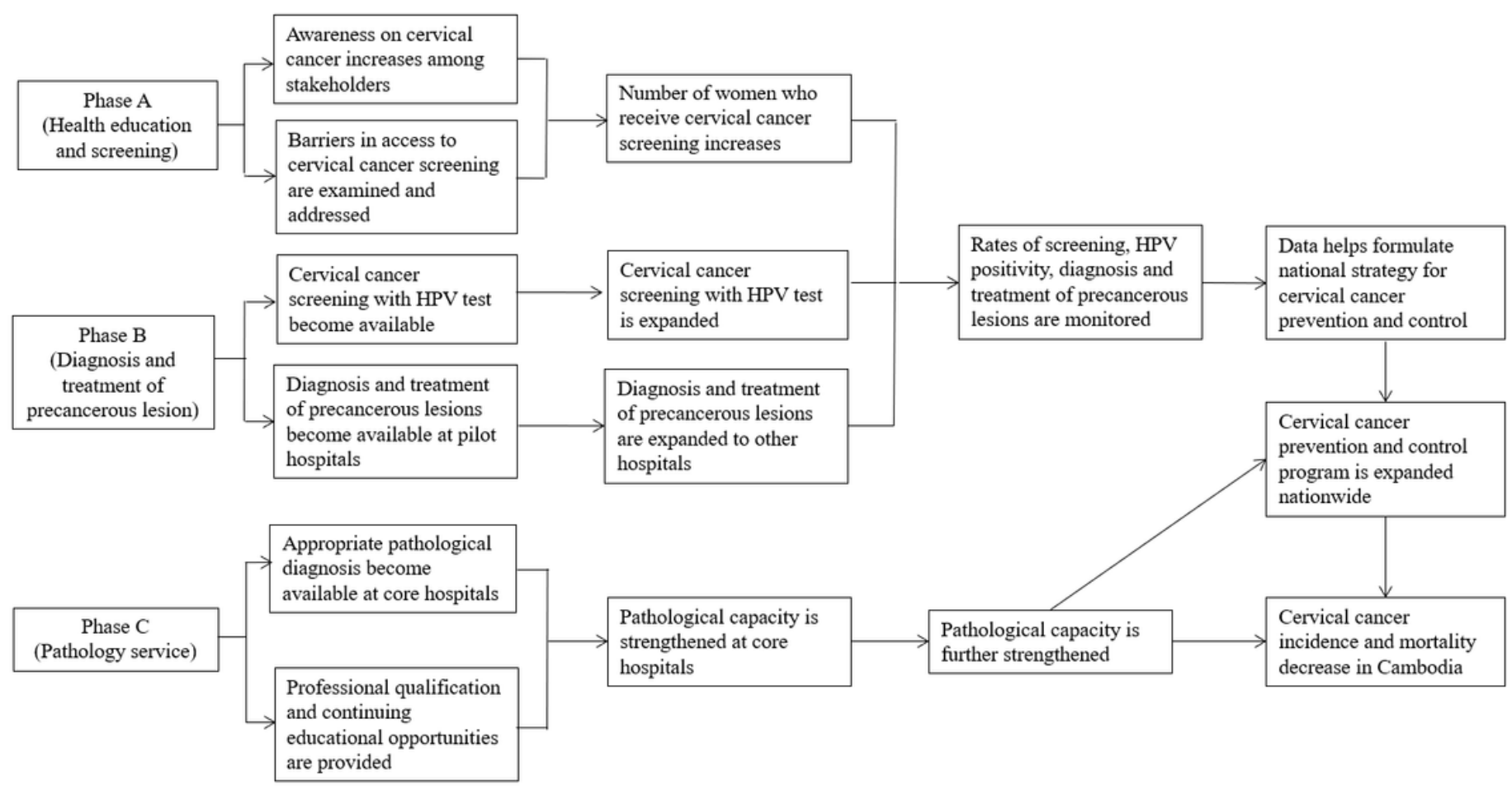

Figure 1

Concept map of the Cambodia Cervical Cancer Project

\section{Supplementary Files}

This is a list of supplementary files associated with this preprint. Click to download.

- Table2.pdf

- Table1.pdf

- Table3.pdf

- Additionalfile1.pdf 